\title{
Synthesis of an arbitrary ABCD system with fixed lens positions
}

\author{
Martin J. Bastiaans \\ Faculteit Elektrotechniek, Technische Universiteit Eindhoven, Postbus 513, 5600 MB Eindhoven, Netherlands \\ Tatiana Alieva \\ Facultad de Ciencias Físicas, Universidad Complutense de Madrid, Ciudad Universitaria s/n, Madrid 28040, Spain
}

Received April 4, 2006; revised June 8, 2006; accepted June 8, 2006; posted June 9, 2006 (Doc. ID 69642); published July 25, 2006

On the basis of the modified Iwasawa decomposition of a lossless first-order optical system as a cascade of a lens, a magnifier, and a so-called orthosymplectic system, we show how to synthesize an arbitrary ABCD system (with two transverse coordinates) by means of lenses and predetermined sections of free space such that the lenses are located at fixed positions. () 2006 Optical Society of America

OCIS codes: $070.2580,070.4690,080.2730,120.4820$.

Any lossless first-order optical system (or ABCD system) can be described by its real symplectic ray transformation matrix ${ }^{1-3} \mathbf{T}$, which relates the position $\vec{r}_{i}$ and direction $\vec{p}_{i}$ of an incoming ray to the position $\vec{r}_{o}$ and direction $\vec{p}_{o}$ of the outgoing ray:

$$
\left[\begin{array}{l}
\vec{r}_{o} \\
\vec{p}_{o}
\end{array}\right]=\mathbf{T}\left[\begin{array}{l}
\vec{r}_{i} \\
\vec{p}_{i}
\end{array}\right]=\left[\begin{array}{ll}
\mathbf{A} & \mathbf{B} \\
\mathbf{C} & \mathbf{D}
\end{array}\right]\left[\begin{array}{l}
\vec{r}_{i} \\
\vec{p}_{i}
\end{array}\right] .
$$

As some well-known one-dimensional (1D) examples we mention the matrices

$$
\left[\begin{array}{cc}
1 & \lambda z \\
0 & 1
\end{array}\right], \quad\left[\begin{array}{cc}
1 & 0 \\
-1 / \lambda f & 1
\end{array}\right], \quad\left[\begin{array}{cc}
0 & w^{2} \\
-w^{-2} & 0
\end{array}\right]
$$

(see Ref. 3, Appendices B.3 and B.4) and in particular ${ }^{4}$

$$
\begin{aligned}
\mathbf{T}_{f}(\theta ; w)= & {\left[\begin{array}{cc}
\cos \theta & w^{2} \sin \theta \\
-w^{-2} \sin \theta & \cos \theta
\end{array}\right]=\left[\begin{array}{cc}
w & 0 \\
0 & w^{-1}
\end{array}\right] } \\
& \times\left[\begin{array}{cc}
\cos \theta & \sin \theta \\
-\sin \theta & \cos \theta
\end{array}\right]\left[\begin{array}{cc}
w^{-1} & 0 \\
0 & w
\end{array}\right],
\end{aligned}
$$

corresponding to a section of free space $\mathcal{S}(z)$ with distance $z$, a lens $\mathcal{L}(f)$ with focal length $f$, a Fourier transformer $\mathcal{F}(\pi / 2 ; w)$ with scaling $w$, and a fractional Fourier transformer ${ }^{4,5} \mathcal{F}(\theta ; w)$ with fractional angle $\theta$ and scaling $w$, respectively, acting on light with wavelength $\lambda$. Usually we work with normalized variables $w^{-1} r$ and $w p$, so that the fractional Fourier transformer $\mathcal{F}(\theta ; w)$ corresponds to a mere rotation in $r p$ space through an angle $\theta$.

Two commonly used coherent-optical realizations of a (1D) fractional Fourier transformer $\mathcal{F}(\theta ; w)$ were suggested by Lohmann ${ }^{4}$ : one setup (a) would consist of a thin (cylindrical) lens $\mathcal{L}(f)$ with focal length $f$, preceded and followed by two identical distances $d$ of free space, $\mathcal{S}(d)$, and the other setup (b) would consist of two identical thin (cylindrical) lenses with focal lengths $f$, separated by a distance $d$. In detail, we have for setup (a), $\mathcal{F}_{a}(\theta ; w)=\mathcal{S}(d) \mathcal{L}(f) \mathcal{S}(d)$,

$$
\begin{aligned}
& \mathbf{T}_{f}(\theta ; w)= {\left[\begin{array}{cc}
1-d / f & \lambda d(2-d / f) \\
-(d / f) / \lambda d & 1-d / f
\end{array}\right], } \\
& w^{2} \tan (\theta / 2)=\lambda d,
\end{aligned}
$$

and for $\operatorname{setup}(\mathrm{b}), \mathcal{F}_{b}(\theta ; w)=\mathcal{L}(f) \mathcal{S}(d) \mathcal{L}(f)$,

$$
\begin{aligned}
\mathbf{T}_{f}(\theta ; w)= & {\left[\begin{array}{cc}
1-d / f & \lambda d \\
-(d / f)(2-d / f) / \lambda d & 1-d / f
\end{array}\right], } \\
& w^{2} \sin \theta=\lambda d,
\end{aligned}
$$

which are equivalent to Eq. (3) for $\sin ^{2}(\theta / 2)=d / 2 f$. Note that in these setups, with $0 \leqslant d / 2 f \leqslant 1$, the fractional angle $\theta$ is restricted to the interval $0 \leqslant \theta \leqslant \pi$, and that for $\theta$ outside that interval we have to perform an additional reversion of the coordinates (or use a cascade of two such fractional Fourier transform setups). Two crossed 1D fractional Fourier transformers, with different fractional angles $\theta_{x, y}$ and different scaling factors $w_{x, y}$, now lead to a twodimensional (2D), separable fractional Fourier transformer $\mathcal{F}\left(\theta_{x}, \theta_{y} ; w_{x}, w_{y}\right)$, with the ray transformation $\operatorname{matrix} \mathbf{T}_{f}\left(\theta_{x}, \theta_{y} ; w_{x}, w_{y}\right)$.

If $d$ is a fixed distance in Lohmann's setups, problems arise when $\theta \approx 0$ or $\theta \approx \pi$, in which case $w^{2}$ may tend to 0 or $\infty$. These problems can be avoided if we restrict $\theta$ to a smaller range around $\pi / 2$, for instance, $-\pi / N \leqslant \theta-\pi / 2 \leqslant \pi / N$ with $N>2$. If we then need a fractional Fourier transformer $\mathcal{F}(\theta ; w)$ with the fractional angle $\theta$ in the full range of length $2 \pi$ (hence $-\pi \leqslant \theta-N \pi / 2 \leqslant \pi$ ), we simply use a cascade of $N$ such reduced-angle transformers, while at the same time avoiding a coordinate reversion.

When we work with normalized coordinates, we will use lowercase characters to denote the resulting normalized matrices. So, with $\mathbf{W}$ a diagonal scaling matrix (to be determined later), we have

$$
\left[\begin{array}{cc}
\mathbf{W}^{-1} & \mathbf{0} \\
\mathbf{0} & \mathbf{W}
\end{array}\right]\left[\begin{array}{l}
\vec{r}_{o} \\
\vec{p}_{o}
\end{array}\right]=\left[\begin{array}{cc}
\mathbf{a} & \mathbf{b} \\
\mathbf{c} & \mathbf{d}
\end{array}\right]\left[\begin{array}{cc}
\mathbf{W}^{-1} & \mathbf{0} \\
\mathbf{0} & \mathbf{W}
\end{array}\right]\left[\begin{array}{l}
\vec{r}_{i} \\
\vec{p}_{i}
\end{array}\right],
$$


where the abcd matrix is the normalized version of the ABCD matrix.

If the ray transformation matrix is not only real symplectic but also orthogonal, we call the system orthosymplectic. ${ }^{6}$ The ray transformation matrix of an orthosymplectic system takes the general form

$$
\left[\begin{array}{cc}
\mathbf{W} & \mathbf{0} \\
\mathbf{0} & \mathbf{W}^{-1}
\end{array}\right]\left[\begin{array}{cc}
\mathbf{x} & \mathbf{y} \\
-\mathbf{y} & \mathbf{x}
\end{array}\right]\left[\begin{array}{cc}
\mathbf{W}^{-1} & \mathbf{0} \\
\mathbf{0} & \mathbf{W}
\end{array}\right],
$$

where $\mathbf{W}$ is again the diagonal scaling matrix and where the two matrices $\mathbf{x}$ and $\mathbf{y}$ can be combined into a complex matrix $\mathbf{u}=\mathbf{x}+i \mathbf{y}$ that is unitary: $\mathbf{u}^{\dagger}=\mathbf{u}^{-1}$, with $\mathbf{u}^{\dagger}$ the transposed and complex conjugated version of $\mathbf{u}$. In the 2D case, with $\vec{r}=\left(r_{x}, r_{y}\right)^{t}$ and $\vec{p}$ $=\left(p_{x}, p_{y}\right)^{t}$, basic members of the orthosymplectic class that we will use in this paper are the $2 \mathrm{D}$ separable fractional Fourier transformer $\mathcal{F}\left(\theta_{x}, \theta_{y} ; w_{x}, w_{y}\right)$ and the rotator $\mathcal{R}(\theta)$ (also called the image gyrator ${ }^{7}$ ), with ray transformation matrices

$$
\begin{aligned}
\mathbf{t}_{f}\left(\theta_{x}, \theta_{y}\right) & =\left[\begin{array}{cccc}
\cos \theta_{x} & 0 & \sin \theta_{x} & 0 \\
0 & \cos \theta_{y} & 0 & \sin \theta_{y} \\
-\sin \theta_{x} & 0 & \cos \theta_{x} & 0 \\
0 & -\sin \theta_{y} & 0 & \cos \theta_{y}
\end{array}\right], \\
\mathbf{t}_{r}(\theta) & =\left[\begin{array}{cccc}
\cos \theta & \sin \theta & 0 & 0 \\
-\sin \theta & \cos \theta & 0 & 0 \\
0 & 0 & \cos \theta & \sin \theta \\
0 & 0 & -\sin \theta & \cos \theta
\end{array}\right],
\end{aligned}
$$

and unitary representations

$$
\begin{aligned}
\mathbf{u}_{f}\left(\theta_{x}, \theta_{y}\right) & =\left[\begin{array}{cc}
\exp \left(i \theta_{x}\right) & 0 \\
0 & \exp \left(i \theta_{y}\right)
\end{array}\right], \\
\mathbf{u}_{r}(\theta) & =\left[\begin{array}{cc}
\cos \theta & \sin \theta \\
-\sin \theta & \cos \theta
\end{array}\right] ;
\end{aligned}
$$

see also Ref. 6, Subsec. 10.3.2, Eq. (10.31), and Ref. 7, Eqs. (49), respectively.

We now represent the normalized symplectic abcd matrix by means of its modified Iwasawa decomposition. ${ }^{8}$ In particular we have (Ref. 6, Subsecs. 9.5 and 10.2)

$$
\left[\begin{array}{ll}
\mathbf{a} & \mathbf{b} \\
\mathbf{c} & \mathbf{d}
\end{array}\right]=\left[\begin{array}{cc}
\mathbf{I} & \mathbf{0} \\
-\mathbf{g} & \mathbf{I}
\end{array}\right]\left[\begin{array}{cc}
\mathbf{s} & \mathbf{0} \\
\mathbf{0} & \mathbf{s}^{-1}
\end{array}\right]\left[\begin{array}{cc}
\mathbf{x} & \mathbf{y} \\
-\mathbf{y} & \mathbf{x}
\end{array}\right],
$$

where the first matrix corresponds to a lens described by the symmetric matrix $\mathbf{G}=\mathbf{W}^{-1} \mathbf{g W}^{-1}$ with $\mathbf{g}$ $=-\left(\mathbf{c a}^{t}+\mathbf{d} \mathbf{b}^{t}\right)\left(\mathbf{a a}^{t}+\mathbf{b b}^{t}\right)^{-1}$, the second matrix corresponds to a magnifier described by the positivedefinite symmetric matrix $\mathbf{S}=\mathbf{W} \mathbf{s W}^{-1}$ with $\mathbf{s}=\left(\mathbf{a a}^{t}\right.$ $\left.+\mathbf{b b}^{t}\right)^{1 / 2}$, and the third matrix represents an orthosymplectic system described by the unitary matrix $\mathbf{u}=\mathbf{x}+i \mathbf{y}=\left(\mathbf{a a}^{t}+\mathbf{b b}^{t}\right)^{-1 / 2}(\mathbf{a}+i \mathbf{b})$.

Based on the modified Iwasawa decomposition, we will now derive how-in the 2D case-an arbitrary ABCD system can be synthesized with fixed lens po- sitions. We remark that (without fixed lens positions) the $1 \mathrm{D}$ case has been treated before, ${ }^{9}$ while an existence proof (without presenting an explicit synthesis method) has been given for the $2 \mathrm{D}$ case. ${ }^{2}$ We recall that we have $\vec{r}=\left(r_{x}, r_{y}\right)^{t}$, that the $4 \times 4$ symplectic ray transformation matrix $\mathbf{T}$ has 10 degrees of freedom, that the matrices $\mathbf{G}, \mathbf{g}, \mathbf{S}, \mathbf{s}, \mathbf{x}$, and $\mathbf{y}$ are $2 \times 2$ matrices, and that the $2 \times 2$ scaling matrix $\mathbf{W}$ still has to be determined. We start with the orthosymplectic subsystem.

It can be shown ${ }^{10}$ that in the $2 \mathrm{D}$ case the orthosymplectic subsystem in the modified Iwasawa decomposition (12), described by the unitary matrix $\mathbf{u}=\mathbf{x}+i \mathbf{y}$, can be realized as a separable fractional Fourier transformer $\mathcal{F}\left(\gamma_{x}, \gamma_{y}\right)$ [see Eqs. (8) and (10)] embedded between two rotators $\mathcal{R}(\alpha)$ and $\mathcal{R}(\beta)$ [see Eqs. (9) and (11)]: $\mathcal{R}(\beta) \mathcal{F}\left(\gamma_{x}, \gamma_{y}\right) \mathcal{R}(\alpha)$. We thus have u $=\mathbf{u}_{r}(\beta) \mathbf{u}_{f}\left(\gamma_{x}, \gamma_{y}\right) \mathbf{u}_{r}(\alpha)$ [see also Ref. 6, Eq. (10.32)]. The fractional angles $\gamma_{x}$ and $\gamma_{y}$ follow from the relations ${ }^{10} \exp \left[i\left(\gamma_{x}+\gamma_{y}\right)\right]=\operatorname{det} \mathbf{u}$ and $\cos \left(\gamma_{x}-\gamma_{y}\right)$ $=\operatorname{det} \mathbf{x}+\operatorname{det} \mathbf{y}$, where the $\pi$ phase ambiguity can be avoided by choosing $0 \leqslant \gamma_{x}-\gamma_{y}<\pi$. The rotation angles $\alpha$ and $\beta$ can then be determined from the elements of the matrices $\mathbf{x}$ and $\mathbf{y} .^{10}$

While the rotators $\mathcal{R}(\alpha)$ and $\mathcal{R}(\beta)$ can be dealt with by simply defining rotated coordinate systems, the separable fractional Fourier transformer $\mathcal{F}\left(\gamma_{x}, \gamma_{y}\right)$ (with different fractional angles $\gamma_{x}$ and $\gamma_{y}$ in the two perpendicular directions) can be realized, for instance, by (cascades of) Lohmann's setups $\mathcal{F}_{a}=\mathcal{S L S}$ or $\mathcal{F}_{b}=\mathcal{L} \mathcal{S L}$, possibly combined with a reversion of the coordinates, as described before. We then have combinations of two crossed cylindrical lenses (with different focal lengths $f_{x}$ and $f_{y}$ ) combined with fixed distances $d$ of free space, with proper rotations of the coordinate systems before and after the separable fractional Fourier transformer. The two fractional angles and the two rotation angles constitute four degrees of freedom.

It is important to note that for any separable fractional Fourier transformer that we want to synthesize, the distance $d$ can be chosen to be constant, which is exactly what we will do; the two focal distances $f_{x}$ and $f_{y}$ are then further determined by $\gamma_{x}$ and $\gamma_{y}$, respectively. The fractional Fourier transformer determines also the scaling factors $w_{x, y}$ (depending on $\sqrt{\lambda d}$ and $\gamma_{x, y}$ ); so the scaling matrix $\mathbf{W}$ is now known.

The lens in the modified Iwasawa decomposition (12) is described by the symmetric matrix $\mathbf{G}$ $=\mathbf{W}^{-1} \mathbf{g W}^{-1}$, which can be decomposed as

$$
\mathbf{G}=\left[\begin{array}{ll}
g_{11} & g_{12} \\
g_{12} & g_{22}
\end{array}\right]=\mathbf{u}_{r}\left(\varphi_{g}\right)\left[\begin{array}{cc}
g_{1} & 0 \\
0 & g_{2}
\end{array}\right] \mathbf{u}_{r}\left(-\varphi_{g}\right),
$$

where $g_{1,2}=\left(g_{11}+g_{22}\right) / 2 \pm\left\{\left[\left(g_{11}-g_{22}\right) / 2\right]^{2}+g_{12}^{2}\right\}^{1 / 2}$ and $\tan \varphi_{g}=\left(g_{11}-g_{1}\right) / g_{12}=g_{12} /\left(g_{22}-g_{1}\right)=g_{12} /\left(g_{2}-g_{11}\right)=\left(g_{2}\right.$ $\left.-g_{22}\right) / g_{12}$ [see also Ref. 6, Subsec. 10.2.1, "Lenses"]. This decomposition shows a possible realization of the 2D (anamorphic) lens as a combination of two crossed cylindrical lenses whose focal lengths read as $1 / \lambda g_{1}$ and $1 / \lambda g_{2}$ and which is oriented at an angle 
$\varphi_{g}: \mathcal{R}\left(\varphi_{g}\right) \mathcal{L}\left(1 / \lambda g_{1}, 1 / \lambda g_{2}\right) \mathcal{R}\left(-\varphi_{g}\right)$. The two focal lengths and the orientation angle constitute three degrees of freedom.

The magnifier in the modified Iwasawa decomposition (12) is described by the positive-definite symmetric matrix $\mathbf{S}=\mathbf{W} \mathbf{s} \mathbf{W}^{-1}$, which can again be decomposed as

$$
\mathbf{S}=\left[\begin{array}{ll}
s_{11} & s_{12} \\
s_{12} & s_{22}
\end{array}\right]=\mathbf{u}_{r}\left(\varphi_{s}\right)\left[\begin{array}{cc}
s_{1} & 0 \\
0 & s_{2}
\end{array}\right] \mathbf{u}_{r}\left(-\varphi_{s}\right)
$$

cf. Eq. (13); see also Ref. 6, Subsec. 10.2.2, "Magnifiers." Note that $s_{1} s_{2}=\operatorname{det} \mathbf{s}>0$ and $s_{1}+s_{2}=\operatorname{Tr} \mathbf{s}>0$. The 2D (anamorphic) magnifier can thus be realized as a combination of two crossed 1D magnifiers whose magnification factors are defined by $s_{1}$ and $s_{2}$ and which is oriented at an angle $\varphi_{s}$.

A 1D magnifier (with reversion) can easily be realized by means of an ideal imaging system built around a thin (cylindrical) lens with focal distance $f_{0}$. The input plane of this system is located a distance $d_{\mathrm{o}}$ before the lens, whereas its output plane is located a distance $z_{0}$ behind the lens. To get ideal imaging, we have of course the condition $1 / d_{0}+1 / z_{0}=1 / f_{0}$, and the magnification is then $s=z_{0} / d_{0}$; to avoid the occurrence of a small value of $f_{\mathrm{o}}$, we require that the magnification $s$ not be too small. To compensate for an unwanted phase factor, we use a thin (cylindrical) phase-correcting lens with focal distance $z_{0}-f_{0}$ in the output plane. In detail we thus have $\mathcal{L}\left(z_{0}\right.$ $\left.-f_{\mathrm{o}}\right) \mathcal{S}\left(z_{\mathrm{o}}\right) \mathcal{L}\left(f_{\mathrm{o}}\right) \mathcal{S}\left(d_{\mathrm{o}}\right)$ :

$$
\begin{gathered}
{\left[\begin{array}{cc}
1 & 0 \\
-1 / \lambda\left(z_{0}-f_{\mathrm{o}}\right) & 1
\end{array}\right]\left[\begin{array}{cc}
1 & \lambda z_{\mathrm{o}} \\
0 & 1
\end{array}\right]\left[\begin{array}{cc}
1 & 0 \\
-1 / \lambda f_{\mathrm{o}} & 1
\end{array}\right]\left[\begin{array}{cc}
1 & \lambda d_{\mathrm{o}} \\
0 & 1
\end{array}\right]} \\
=-\left[\begin{array}{cc}
\left(z_{\mathrm{o}}-f_{\mathrm{o}}\right) / f_{\mathrm{o}} & 0 \\
0 & f_{\mathrm{o}} /\left(z_{\mathrm{o}}-f_{\mathrm{o}}\right)
\end{array}\right] \equiv-\left[\begin{array}{cc}
s & 0 \\
0 & s^{-1}
\end{array}\right] .
\end{gathered}
$$

Note that the overall minus sign corresponds to reversion; we will compensate for that later.

Two crossed 1D magnifiers, with different magnifications $s_{1}=z_{1} / d_{1}$ and $s_{2}=z_{2} / d_{2}$ in the two perpendicular directions, and with $z_{1}+d_{1}=z_{2}+d_{2}$, lead to a $2 \mathrm{D}$ separable magnifier. The two magnification factors and the orientation angle again constitute three degrees of freedom.

To obtain fixed lens positions in the magnifier (and thus choosing $d_{1}=d_{2}=d_{0}$ ), we remark that a (1D) section of free space $z_{0}=s d_{0}$ can be synthesized by a lens embedded between two Fourier transformers, $\mathcal{S}\left(z_{0}\right)$ $=\mathcal{F}(\pi / 2 ; w) \mathcal{L}(f) \mathcal{F}(\pi / 2 ; w)$ :

$$
\begin{aligned}
& {\left[\begin{array}{cc}
0 & w^{2} \\
-w^{-2} & 0
\end{array}\right]\left[\begin{array}{cc}
1 & 0 \\
-1 / \lambda f & 1
\end{array}\right]\left[\begin{array}{cc}
0 & w^{2} \\
-w^{-2} & 0
\end{array}\right]=-\left[\begin{array}{cc}
1 & w^{4} / \lambda f \\
0 & 1
\end{array}\right]} \\
& \equiv-\left[\begin{array}{cc}
1 & \lambda z_{0} \\
0 & 1
\end{array}\right]
\end{aligned}
$$

The distance $z_{0}$, the focal distance $f$ of the lens, and the scaling $w$ of the Fourier transformers are related as $f=w^{4} / \lambda^{2} z_{0}$; to avoid a small value of $f$, we now also require that $s$ not be too large. Note that the coordinate reversion that corresponds to the overall minus sign in Eq. (16) compensates for the reversion that we encountered before in Eq. (15). A (1D) magnifier with magnification $s=z_{0} / d_{0}$ can thus be synthesized by a cascade of a section of free space with constant distance $d_{0}$, a lens with focal distance $f_{0}=\left(1 / d_{0}\right.$ $\left.+1 / z_{0}\right)^{-1}$, a lens with focal distance $w^{4} / \lambda^{2} z_{0}$ embedded between two Fourier transformers with scaling $w$, and a phase-correcting lens with focal distance $z_{0}$ $-f_{\mathrm{o}}: \mathcal{L}\left(z_{\mathrm{o}}-f_{\mathrm{o}}\right) \mathcal{F}(\pi / 2 ; w) \mathcal{L}\left(w^{4} / \lambda^{2} z_{\mathrm{o}}\right) \mathcal{F}(\pi / 2 ; w) \mathcal{L}\left(f_{\mathrm{o}}\right) \mathcal{S}\left(d_{\mathrm{o}}\right)$.

The final $2 \mathrm{D}$ cascade then takes the form

$$
\begin{aligned}
& \mathcal{R}\left(\varphi_{g}\right) \mathcal{L}\left(1 / \lambda g_{1}, 1 / \lambda g_{2}\right) \mathcal{R}\left(-\varphi_{g}\right) \mathcal{R}\left(\varphi_{s}\right) \mathcal{L}\left(z_{1}-f_{1}, z_{2}-f_{2}\right) \\
& \quad \times \mathcal{F}_{b}(\pi / 2 ; w) \mathcal{L}\left(w^{4} / \lambda^{2} z_{1}, w^{4} / \lambda^{2} z_{2}\right) \mathcal{F}_{b}(\pi / 2 ; w) \\
& \quad \times \mathcal{L}\left(f_{1}, f_{2}\right) \mathcal{S}\left(d_{o}\right) \mathcal{R}\left(-\varphi_{s}\right) \mathcal{R}(\beta) \mathcal{F}\left(\gamma_{x}, \gamma_{y} ; w_{x}, w_{y}\right) \mathcal{R}(\alpha) .
\end{aligned}
$$

Besides the constant distance $d_{0}$, sections of free space appear only inside the (fractional) Fourier transformers and can be chosen to be constant, resulting in fixed positions of the lenses. Of course, rotators $\mathcal{R}$ and sections of free space $\mathcal{S}$ commute, and adjacent rotators $\mathcal{R}$ and lenses $\mathcal{L}$ can be combined into one single (anamorphic) lens with a proper orientation, in which case the number of lenses (and predetermined sections of free space) in the final cascade (17), $\mathcal{R} \mathcal{L} \mathcal{R} \mathcal{R} \mathcal{L} \mathcal{L} \mathcal{S} \mathcal{L} \mathcal{L} \mathcal{L} \mathcal{S} \mathcal{L} \mathcal{S} R \mathcal{R} \mathcal{F} \mathcal{R}$, is reduced to three plus the number of lenses used to realize the fractional Fourier transformer $\mathcal{F}\left(\gamma_{x}, \gamma_{y} ; w_{x}, w_{y}\right)$.

T. Alieva thanks the Spanish Ministry of Education and Science for financial support (TEC2005-02180/ MIC). M. J. Bastiaans can be reached at m.j.bastiaans@tue.nl, and T. Alieva at talieva@fis.ucm.es.

\section{References}

1. R. K. Luneburg, Mathematical Theory of Optics (U. California Press, 1966).

2. E. C. G. Sudarshan, N. Mukunda, and R. Simon, Opt. Acta 32, 855 (1985).

3. J. W. Goodman, Introduction to Fourier Optics, 2nd ed. (McGraw-Hill, 1996).

4. A. W. Lohmann, J. Opt. Soc. Am. A 10, 2181 (1993).

5. H. M. Ozaktas, Z. Zalevsky, and M. A. Kutay, The Fractional Fourier Transform with Applications in Optics and Signal Processing (Wiley, 2001).

6. K. B. Wolf, Geometric Optics on Phase Space (Springer, 2004).

7. R. Simon and K. B. Wolf, J. Opt. Soc. Am. A 17, 342 (2000).

8. R. Simon and N. Mukunda, J. Opt. Soc. Am. A 15, 2146 (1998).

9. M. Nazarathy and J. Shamir, J. Opt. Soc. Am. 72, 356 (1982).

10. T. Alieva and M. J. Bastiaans, Opt. Lett. 30, 3302 (2005). 